\title{
Direct and Mediated Impacts of Product and Process Characteristics on Consumers' Choice of Organic vs. Conventional Chicken
}

\section{Livia Marian ${ }^{*} \&$ John Thøgersen ${ }^{a}$}

${ }^{\text {a }}$ Aarhus University, School of Business and Social Sciences, MAPP, Department of Business Administration, Bartholins Allé 10, Aarhus C, Denmark

*Corresponding author: Aarhus University, School of Business and Social Sciences, MAPP, Department of Business Administration, Bartholins Allé 10, Aarhus C, Denmark. Tel.: +45 8716 01 95, E-mail: livma@asb.dk 


\begin{abstract}
There is a lack of research into why consumers value process characteristics. In this study, we test the hypothesis that the impact of process characteristics such as organic and free-range on consumers' choices of food products is at least partly mediated through expected eating quality or taste expectations. In other words, the process characteristics partly function as cues to (eating) quality. Using a traditional metric conjoint approach based on an additive model, four product characteristics (production method, price, size and information about farmer and rearing conditions) were varied in a fractional factorial conjoint design, creating nine profiles of whole chickens. 384 respondents rated the nine different chickens in terms of taste expectations and willingness to buy. Since the nine records for each respondent are not independent, we used linear mixed modelling for the mediation analysis. We find that, as expected, taste expectations are a strong predictor of willingness to buy. As hypothesized, the impact of both product and process characteristics on willingness to buy is at least partly mediated through taste expectations. Hence, the study shows that process characteristics are important for consumers, not only in and off themselves, but partly because consumers make inferences about eating quality from knowledge about such process characteristics.
\end{abstract}

Keywords: consumer choice, quality cues, expected taste, conjoint analysis, mediation analysis, organic chicken 


\section{Introduction}

At a general level, consumer food choices are determined by three major factors: the food, the environment and the individual (Conner \& Armitage, 2002). As regards the food itself, its physical composition determines the properties that are perceived as sensory characteristics, including its taste. In the purchase situation, the consumer rarely knows the exact quality of offered food products, but forms expectations about quality based on available cues and the decision to buy is made based on these expectations. Different cues to quality may be available to different consumers in different situations and they may differ in their predictive validity and power (Becker, 2000; Bruns $\varnothing$, Fjord, \& Grunert, 2002). Consumers may even be aware that some of the cues they use to form expectations about attributes, such as taste or tenderness, are not particularly predictive in all cases (Grunert, 2005). Only after the purchase of a food product, the consumer has the chance to experience its quality, which may or may not correspond to the expected quality. The quality of a food product is evaluated based on many dimensions, but sensorial experiences play a key role, especially taste (Acebron \& Dopico, 2000; Brunsø, et al., 2002).

A large number of studies of the importance of taste in food choice have been published. Qualitative studies of the values underlying people's attitudes find that taste is an important experience characteristic, quality criterion and purchase motivator (Bredahl \& Poulsen, 2002; Van Loo et al., 2010). Studies of organic food find that taste expectations are higher than for conventional food (Kennedy, Stewart-Knox, Mitchell, \& Thurnham, 2004; Naspetti \& Zanoli, 2009; Stolz, Jahrl, Baumgart, \& Schneider, 2010; Zanoli \& Naspetti, 2002). Similar results are found in survey studies. For example, one study found that $87 \%$ of a sample of Greek consumers considers taste to be the most important reason for their meat preferences (Krystallis \& Arvanitoyannis, 2006) and another study reports that for 56\% of Greek buyers better taste is the main reason for their organic food preference (Krystallis, Arvanitoyannis, \& Chryssohoidis, 2006).

These studies, together with studies of the cues consumers use to infer the quality of a food product (Becker, 2000; Bruns $\varnothing$, Fjord, \& Grunert, 2002), raise the question whether consumers value specific product and process characteristics in their own right or because they use them as cues to eating quality and to form expectations about the pleasure derived from eating the product? However, no previous study has investigated if and how consumers' quality inferences mediate the influence of product and process characteristics on product choice.

In this study we test the hypothesis that the impact of important product and process characteristics on consumers' choice of food products is at least partly mediated through expected eating quality or taste expectations. We use whole chicken as the product of study. It is well documented that consumers often select meat products based on the expected sensorial experience and taste, and that these expectations are based on the available visual cues (Krystallis \& Arvanitoyannis, 2006; Van Loo, et al., 2010). Moreover, meat products cannot be evaluated completely until they are finally prepared, in a post-purchase phase (Acebron \& Dopico, 2000), which means that consumers need to rely on the information they get in the pre-purchase phase as cues to quality. We analyse both the direct effects of specific product and process characteristics on the choice of whole chicken and the indirect effect mediated through expected taste.

\subsection{Prior research}

Food choices are seemingly simple choices, which consumers deal with frequently and effectively, also when the chosen products contain highly involving attributes (Thøgersen, Jørgensen, \& Sandager, 2012). However, food choices are more complex than it may seem from a superficial 
observation. Consumer responses to a given food can be divided into four classes: sensory, affective, cognitive and behavioural (Costell, Tárrega, \& Bayarri, 2010). Food choice is to a high extent a learned behaviour and an individual's liking of a specific food product depends on the frequency of the person's previous exposure to that product (Köster \& Mojet, 2007).

Based on Bandura's (1991, 1998) social-cognitive theory, Conner and Armitage (2002) suggest that food choices are determined by three major factors: the food, the environment and the individual. The food itself affects food choice through different routes, including sensorial effects, such as taste, appearance and smell. However, it is not the sensory characteristics in themselves that influence food choice; it is the preference for certain combinations of characteristics in diverse eating situations (Conner \& Armitage, 2002). Consumers seek food with certain sensory characteristics, which is why sensory quality is a key determinant of food acceptance and choice (Costell, et al., 2010). Research unanimously points at expected taste as an important quality dimension in food choice (Bredahl \& Poulsen, 2002; Kennedy, et al., 2004; Krystallis \& Arvanitoyannis, 2006; Stanton, Hughner, McDonagh, Prothero, \& Shultz, 2007; Stolz, et al., 2010; Van Loo, et al., 2010).

Consumers evaluate quality both at the point of purchase and at the point of consumption. Quality expectations are formed on the basis of intrinsic as well as extrinsic quality cues. Intrinsic cues are attributes that are built-in to the product itself (e.g., size, colour), whereas extrinsic cues are those characteristics that can be modified without influencing the product directly (e.g., price, packaging, the type of retail outlet) (Veale \& Quester, 2009). The importance of intrinsic attributes at the point of purchase depends on whether they can be evaluated at that time and used as quality indicators (Zeithaml, 1988).

Previous research shows that, overall, intrinsic cues are typically given more credence than extrinsic cues. Extrinsic cues are used mostly when intrinsic cues are not predictive enough or when consumers have little confidence in their ability to evaluate and assess them (Veale \& Quester, 2009). The less the predictive value of the intrinsic cues, the higher the importance of extrinsic cues (Acebron \& Dopico, 2000; Becker, 2000).

Product attributes that can be ascertained prior to consumption - be they intrinsic or extrinsic - can be used as quality cues by consumers. The taste of a product cannot usually be evaluated before consumption. Hence, consumers use available cues to predict the taste of food products in the choice situation (Northen, 2000). Consumers' quality perceptions depend to a large extent on the predictability and availability of quality cues (Bruns $\varnothing$, et al., 2002). The less the predictive value of the cues, the less consumers are able to select the quality they want (Becker, 2000). Consumers prefer cues that they believe to be predictive of the desired quality, but also cues that they feel confident in using, even though they actually are not highly predictive. Inference-making in the quality perception process is an enigmatic area of consumer behaviour, which is tightly connected to the knowledge and expertise of the consumer (Grunert, 2005).

According to the Total Food Quality Model, the intention to buy a certain product is co-determined by perceived costs and expected quality (Bruns $\varnothing$, et al., 2002). Expected, as well as experienced, food quality has four major dimensions, which appear to be universal: taste and appearance, health, convenience and process. Expected taste plays a major role in determining food choices, while experienced taste is an important quality evaluator in the post-purchase phase, even though the concrete taste experience is quite subjective (Aertsens, Verbeke, Mondelaers, \& Van Huylenbroeck, 
2009). Indeed, a food product's taste can usually only be established after consumption and thus represents an experience characteristic of a food product. Several studies have shown that taste is important when it comes to organic food as well; some concluding that in this case consumers have even higher taste expectations (Aertsens, et al., 2009; Krystallis, et al., 2006; Magnusson et al., 2001; McEachern \& Willock, 2004; Naspetti \& Zanoli, 2009; Scholderer, Nielsen, Bredahl, CaludiMagnussen, \& Lindahl, 2004; Stanton, et al., 2007; Thøgersen, 2009; Van Loo, et al., 2010; Zanoli \& Naspetti, 2002).

In recent years, consumers have attached increasing importance to attributes that have no necessary relationship to the experienced product quality. Especially the way food is produced has become an important quality dimension, even in cases when it has no immediate bearing on the taste of the product (Bruns $\varnothing$, et al., 2002).

\section{Methods}

\subsection{Focus groups}

In order to determine what Danish consumers perceive to be the most important quality cues and quality dimensions for chicken products in general, and for organic chicken in particular, two focus group interviews $(n=7 ; n=8)$ were carried out. The attributes that were identified as most important in the focus groups were used to create the conjoint profiles in the second phase of the study.

According to the focus groups, taste is the most important quality dimension for chicken products, which suggests that sensorial characteristics are an important determinant of consumers' overall evaluation of chicken products. When comparing conventional and organic chicken, participants in the focus group did not assess the taste of organic chicken as necessarily better than the taste of conventional chicken. In addition, the focus groups revealed that the most important extrinsic quality cues are price, date stamp, origin, information about the farmer and the rearing conditions, and organic and free-range labels. Major intrinsic quality cues include size, freshness (whether the product is fresh or frozen), appearance and colour. Participants perceive low price and low weight as cues indicating low quality, whereas organic, fresh, whole chicken and a high price are all cues indicating high quality.

\subsection{Conjoint design}

The second part of the study comprises a questionnaire-based survey with a convenience sample of ordinary chicken consumers. Participants were first recruited in two supermarkets in Aarhus having a high share of organic groceries. Customers who agreed to participate received the questionnaire by e-mail. In addition, questionnaires were distributed on-line on social network platforms, using the snowball technique, including the webpage okologi.org and the Facebook pages of Organic Denmark, I Love ØKO and Aarhus Økologiske Fødevarefællesskab. Additionally, the questionnaire was distributed to personal contacts. A total of 405 individuals agreed to participate in the survey. After screening away those that did not eat chicken or did not have any influence on the household's grocery shopping, as well as a few due to odd response patterns (e.g., giving the same score to all conjoint profiles), the final sample consisted of 384 respondents.

The conjoint analysis design, which is used for the present purpose, was embedded in a larger questionnaire containing, among others, questions about demographic characteristics and various behavioural and attitudinal questions. The conjoint analysis task was based on illustrations of whole 
fresh chickens. An orthogonal conjoint design containing nine profiles was developed based on four of the most important attributes identified in the focus groups: size, price, production method and information about the farmer and the rearing conditions, see Table 1.

Table 1: Whole chicken attributes and attribute levels

\begin{tabular}{lll}
\hline \multicolumn{1}{c}{ Attribute } & Levels & Cue Type \\
\hline Size & $1.2 \mathrm{~kg}$ & Intrinsic \\
& $1.6 \mathrm{~kg}$ & \\
& $2.0 \mathrm{~kg}$ & Extrinsic \\
& $40 \mathrm{kr} / \mathrm{kg}$ & \\
& $67.5 \mathrm{kr} / \mathrm{kg}$ & \\
& $95 \mathrm{kr} / \mathrm{kg}$ & Extrinsic \\
\hline Price & Conventional & \\
& Free-range & Extrinsic \\
\hline Information about the farmer & Organic & Information \\
and the rearing conditions & No information & \\
\hline
\end{tabular}

Size had three levels: $1.2 \mathrm{~kg}, 1.6 \mathrm{~kg}$ and $2 \mathrm{~kg}$. The results from the focus groups showed that these levels correspond to consumers' perceptions of small, medium and large-sized chickens. An in-store investigation confirmed that these figures reflect the range of commonly found weights of whole chicken in Danish stores.

Price per $\mathrm{kg}$ had three levels: $40 \mathrm{kr} ., 67.5 \mathrm{kr}$. and $95 \mathrm{kr}$. The minimum and the maximum levels were determined based on in-store observations and reflect the actual market prices of whole chicken. $40 \mathrm{kr}$. represents the standard price for a whole fresh conventional chicken, whereas $95 \mathrm{kr}$. is the average price consumers have to pay for a whole organic chicken. The middle level of 67.5 $\mathrm{kr}$. was chosen based on the recommendation to have an equal range between numeral levels in a conjoint design.

Production method also had three levels: organic, free-range and conventional.

The presence or absence of information about the farmer and the rearing conditions on the label resulted in two levels for this attribute.

A full factorial design generates $54(3 * 3 * 3 * 2)$ different profiles. Given the fact that it would be too taxing for the respondents to evaluate all the 54 possibilities, an orthogonal fractional factorial design was generated using SPSS, which resulted in 9 different whole chicken profiles. The respondents were presented with illustrations of the 9 chicken profiles and were asked to rate their taste expectation and their willingness to buy each of the 9 different chicken (in that order). The ratings were given on eleven-point bi-polar scales, with 0 representing "very bad taste" and "not at all willing to buy" and 10 representing "very good taste" and "very willing to buy". Due to the fact that each respondent was presented with all the 9 different profiles and rated them successively, the individual chicken ratings are not independent, which has implications for the statistical analysis of the data.

The fact that participants rated both expected taste and their willingness to buy made it possible to conduct two different conjoint analyses with the attribute levels (i.e., quality cues) as initial 
variables and expected taste, respectively willingness to buy, as outcome variables. We integrated the analysis of these two evaluations when testing the hypothesis that the impact of quality dimensions on consumers' willingness to buy a specific chicken (e.g., organic vs. conventional or free-range) is partly mediated through their taste expectations. In the mediational model, quality cues are initial variables, willingness to buy is the outcome variable and expected taste is the mediator.

\subsection{Mediation analysis}

In order to better understand the mechanisms through which product characteristics affect buying intentions, in this case for whole chicken products, we test the possible mediation of the effects of product characteristics (the initial variables) on consumer buying intention (the outcome) through expected taste (the mediator). A mediational model is a causal model, meaning that product characteristics are presumed to cause expected taste, which is presumed to cause buying intention, alone or together with product characteristics. Because we manipulated product characteristics in our experimental design, we can be sure that the causality assumption is correct with regard to the initial variable. We asked participants to assess the expected taste of each chicken before they were asked to report their buying intention for the same chicken, which makes it more likely that the former influenced the latter than vice versa. However, our study design does not guarantee that this causal assumption is correct. This reservation should be kept in mind when interpreting the results.

In the mediation analysis, we follow the four steps proposed by Baron and Kenny (1986):

Step 1: Establish that there is an effect that may be mediated by showing that the initial variable is significantly related to the outcome.

Step 2: Show that the initial variable is significantly related to the mediator.

Step 3: Show that the mediator affects the outcome variable while controlling for the initial variable.

Step 4: To establish that the mediator completely mediates the relationship between the initial variable and the outcome, the effect of the initial variable on the outcome while controlling for the mediator should be zero. If the path from the initial variable to the outcome is reduced in absolute size, but is still different from zero when the mediator is introduced, this is a case of partial mediation.

Hence, if the first three steps are met, the data are consistent with the hypothesis that the mediator at least partially mediates the relationship between the initial variable and the outcome. However, only if it is possible to rule out other models that are consistent with the data can one conclusively establish that mediation has occurred.

At his mediation website, ${ }^{1}$ Kenny cautions that the steps should be stated in terms of zero and nonzero coefficients rather than statistical significance, because trivially small coefficients can be statistically significant with large sample sizes and very large coefficients can be non-significant with small sample sizes. In the case of partial mediation, the total effect of the initial variable on the outcome is divided into a direct effect and a mediated or indirect effect. The indirect effect is the product of the effect of the initial variable on the mediator and the effect of the mediator on the outcome. Contemporary mediational analyses focus mostly on the indirect effect.

\footnotetext{
${ }^{1}$ http://davidakenny.net/cm/mediate.htm\#IE (accessed 13 April 2012)
} 


\subsection{Linear Mixed Modelling}

Since our data contain observations that are not independent, we perform the mediation analysis by means of linear mixed modelling (LMM) using SPSS19. For this analysis, we organized our data in the multiple record (MR) format. The MR format means that each participant is represented by as many rows as there are rated product descriptions (i.e., 9). Hence, measures at the individual product description level are at level 1 and they are nested within participants at level 2. In other words, participants are the grouping variable each delivering nine assessments of expected taste and nine buying intentions. Because each participant evaluated nine product variants, there is a clustering of effects in their responses, which leads to correlated errors. This lack of independence is handled by including the participant ID as a random factor in the model. Specifically, we modelled the studied relationships as a two-level random intercept model, meaning that the intercept of the level 1 dependent is modelled as an effect of the level 2 grouping variable (and of the level 1 covariates).

In the service of parsimony and to avoid excess complexity of the modeling, we made some simplifying assumptions. First, and because it is sufficient for our present purpose to estimate the central tendency of the effects of independent variables in the population, the initial variables (i.e., product attributes, coded as dummy variables) are treated as fixed effects in the model as is expected taste when included as a mediator. This implies that the effects of independent variables (other than the participant ID) are assumed to be constant across participants. ${ }^{2}$ Second, and because the various chicken profiles were presented in no particular order, we specified the covariance structure for the "repeated measurement" to be of the variance component type. This is the simplest type and also the default specification in SPSS. It implies that all individual response tendencies are captured by the participant ID, which is satisfactory for the present purpose.

In the following, we sometimes compare the model of interest to a reference model called "the null model," which is a random intercept model without level 1 covariates. Hence, it only includes the participant ID as independent variable: the level 1 intercept is predicted as a random effect of the level 2 grouping variable (i.e., participants).

\section{Results}

\subsection{Mediation analysis}

We use the likelihood ratio test to test the steps in the mediation analysis. When fitting a LMM in SPSS, the -2 Log Likelihood (-2LL) is among the fit measures reported in the output. This makes it possible to measure the contribution of a predictor term by the difference between the -2LL coefficients of a model with and one without the term in question; commonly referred to as a chisquare difference. The degrees of freedom (df) are the difference in df between the two models, which is equal to the difference in the number of terms in the two models. If the term in question is a fixed effect, as in our case, ML estimation is used for the likelihood ratio test.

\footnotetext{
${ }^{2}$ If we wanted to relax this assumption, we could have applied a random effects model instead, which would make it possible to estimate variances of the effects of the independent variables. However, for the present purpose the small gain in the precision of our conclusions from applying this procedure, which would have no substantial implications for assessing the central tendency, does not | justify the increased complexity of the statistical analysis, in our view.
} 


\begin{tabular}{|c|c|c|c|c|c|c|}
\hline & & $-2 \mathrm{LL}$ & Parameters & $\Delta(-2 \mathrm{LL})$ & df. & Sign. \\
\hline & Buying intention & & & & & \multirow[b]{3}{*}{$<.001$} \\
\hline 1 & Null model & 17711 & 3 & & & \\
\hline 2 & Product attributes & 16450 & 10 & 1261 & 7 & \\
\hline 3 & Expected taste & 16182 & 4 & 1529 & 1 & $<.001$ \\
\hline 4 & $\begin{array}{l}\text { Product attributes and } \\
\text { expected taste }\end{array}$ & 15469 & 11 & 981 & 1 & $<.001$ \\
\hline & Expected taste & & & & & \\
\hline 5 & Null model & 15153 & 3 & & & \multirow[b]{2}{*}{$<.001$} \\
\hline 6 & Product attributes & 13194 & 10 & 1960 & 7 & \\
\hline
\end{tabular}

The deviance (-2 LL) of the random intercept model with product attributes (the initial variables) as level 1 covariates and buying intention as the dependent variable is 16450 compared to 17711 for the null model (Table 2). Hence, the former model has a better fit being 1261 lower than the null model. With $7 \mathrm{df}$, this chi-square difference is highly significant. Hence, the product attributes model is different from and better than the null model at a significance level better than .001 . This likelihood ratio test shows that the initial variables are significantly related to the outcome and thereby establishes that there is an effect that may be mediated (Step 1).

In the same way, we see that the difference in fit between the null model and the random intercept model with product attributes as level 1 covariates and expected taste as the dependent variable is highly significant (chi-square difference $=1960,7 \mathrm{df}, \mathrm{p}<.001$ ). This shows that the initial variables are significantly related to the mediator (Step 2).

Finally, we see that the difference in fit between the product attributes model and the model with both product attributes and expected taste as level 1 covariates and buying intention as the dependent variable is highly significant (chi-square difference $=981,1 \mathrm{df}, \mathrm{p}<.001$ ). This shows that the mediator affects the outcome variable while controlling for the initial variables (Step 3). In order to establish whether the mediator completely or only partially mediates the relationship between the initial variables and the outcome (Step 4), we will now inspect the regression coefficients in these two models. 
Table 3: Estimates of fixed effects using buying intentions as dependent variable

\begin{tabular}{|c|c|c|c|c|c|c|}
\hline & \multicolumn{3}{|c|}{ Model 1} & \multicolumn{2}{|c|}{ Model 2} & \multirow[b]{2}{*}{ Sig. } \\
\hline & $\mathrm{B}$ & $\mathrm{t}$ & Sig. & $\mathrm{B}$ & $\mathrm{t}$ & \\
\hline Intercept & 4.47 & 31.408 & $<.001$ & 5.98 & 43.861 & $<.001$ \\
\hline Low price & 0.95 & 9.384 & $<.001$ & 1.11 & 12.945 & $<.001$ \\
\hline High price & -1.11 & -11.001 & $<.001$ & -1.14 & -13.277 & $<.001$ \\
\hline Low weight & -0.26 & -2.580 & .010 & -0.12 & -1.432 & .152 \\
\hline High weight & -0.23 & -2.262 & .024 & -0.34 & -3.948 & $<.001$ \\
\hline Organic & 3.33 & 32.976 & $<.001$ & 0.88 & 7.877 & $<.001$ \\
\hline Free range & 2.01 & 19.954 & $<.001$ & 0.31 & 3.078 & .002 \\
\hline Information & 0.49 & 5.562 & $<.001$ & 0.22 & 2.885 & .004 \\
\hline $\begin{array}{l}\text { Expected } \\
\text { taste }\end{array}$ & & & & 0.80 & 34.023 & $<.001$ \\
\hline
\end{tabular}

The fixed effects parameters in a LMM can be interpreted as unstandardized regression coefficients in the same way as in ordinary regression. The intercept is interpreted as the overall mean of the dependent variable when all independent variables are zero. For example, the parameter estimate for expected taste is 0.8 (Table 3, Model 2), which means that for a unit increase in expected taste, buying intention (the dependent variable) will increase 0.8 units. If the fixed effect is a $0-1$ dichotomy, such as non-organic $=0$, organic $=1$, then a coefficient of 3.33 (Table 3, Model 1) means that the average buying intention is 3.33 units higher for an organic than for a non-organic chicken, everything else being equal.

In Model 1, we see that all included product attributes (initial variables) have a significant effect on buying intentions. Both organic and free range are apparently strongly favoured over conventional chicken and both product characteristics lead to a significant and substantial increase in buying intentions, and substantially bigger for organic than for free range. Price has the expected (approximately linear) negative effect on buying intentions. Information about the farmer has a positive, but relatively weak effect on buying intentions. Low weight and high weight both have a negative impact on buying intentions, compared to medium weight. Apparently these consumers' ideal chicken has a medium weight.

When the presumed mediator is included in the analysis, in Model 2, we first note that it has a highly significant effect on the dependent variable, as already established in the likelihood ratio test. Next, notice that several fixed effects parameters (B) are substantially attenuated compared to Model 1 and the negative impact of low weight is no longer significant. This suggests that the effect of low weight on buying intentions is completely mediated through expected taste, whereas the effects of organic, free range and information about the farmer are partially mediated. The effects of price and high weight are apparently not mediated through expected taste. However, before drawing that conclusion, we need to inspect the effects of product attributes on expected taste (Table 4). 
Table 4: Estimates of fixed effects using expected taste as dependent variable

\begin{tabular}{llll}
\hline & $\mathrm{B}$ & $\mathrm{t}$ & Sig. \\
\hline Intercept & 6.18 & 62.930 & $<.001$ \\
Low price & -0.21 & -3.446 & $<.001$ \\
High price & 0.04 & 0.643 & .520 \\
Low weight & -0.17 & -2.817 & .005 \\
High weight & 0.14 & 2.288 & .022 \\
Organic & 3.05 & 50.230 & $<.001$ \\
Free range & 2.13 & 35.074 & $<.001$ \\
Information & 0.34 & 6.406 & $<.001$ \\
\hline
\end{tabular}

It appears from Table 4 that in all the cases where effects of product attributes (the initial variables) were substantially attenuated after controlling for expected taste (the moderator) in Table 3 , these product attributes have the expected significant impact on expected taste. Consumers indeed seem to infer expectations about a chicken's taste from its weight (a low-weight chicken is expected to have inferior taste qualities), as well as from information that the chicken is either organic or free range or just information about the farmer (all of which are apparently used as cues indicating superior taste qualities). This is consistent with the hypothesis that the impact of these product attributes on consumer buying intentions is completely (low weight) or partly (all other included attributes) mediated through expected taste. Hence, we may infer that an important reason why consumers prefer chicken that are not too small, that are organic or free range, and that come with information about the farmer is that they use all of these attributes as cues to the taste quality of the chicken.

The effects of organic, free range and information about the farmer on buying intentions are only partially mediated through expected taste. Hence, consumers not only prefer a chicken with these attributes because they are perceived as indicators of superior taste. Arguably, each of these attributes represents something that many consumers favour in their own right. However, we note that in each of these three cases the direct effect (Table 3, Model 2) is less than half of the total effect (Table 3, Model 1) of the attribute on buying intentions. For organic, the direct effect is $26 \%$ and for free range a mere $15 \%$ of the total effect.

The direct effect after controlling for taste also suggests that consumers to some extent make tradeoffs and are willing to sacrifice taste for organic or free range and vice-versa. However, the strong mediation effect shows that process characteristics such as organic and free range are not independent from expected taste, which limits the trade-offs in practice.

Notice also that the mediation analysis also adds some nuances to the understanding of the impact of high weight and price on buying intentions. According to Table 4, a larger than average chicken is expected to taste better than average and a low-price chicken to taste worse. The positive effect of high weight and the negative effect of a low price are opposite to the effects on buying intention that appears in Table 3. Hence, these are examples of what mediation analysis calls inconsistent mediation (MacKinnon, Fairchild, \& Fritz, 2007). When there is inconsistent mediation, the 
mediator acts like a suppressor variable, which is why the direct effects of high weight and low price (Table 3, Model 2) are larger than their total effect (Table 3, Model 1).

\subsection{Explained variance}

The SPSS LMM output contains estimates of two variance components: between "groups" (i.e., participants in our case) and residual variation. The residual component is the variance in buying intentions (Table 3) or expected taste (Table 4) not explained by the between-participants effect and hence represents variation within participants, across the nine products evaluated by each participant. Hence, we refer to this as the "within-participants" variance. The between-participants variance is the estimated variance of the intercepts between participants. Among other things, these two variance components reveal whether the variation in buying intentions is primarily within or between participants. The total variance is the sum of the between- and within-participants variance.

In the null model for buying intention, where only the participant ID is included in the equation (as a random factor), the between-participants variance component is 1.59 (Wald $\mathrm{Z}=8.495, \mathrm{p}<.001$ ). The within-participant variance component estimate is 8.84 (Wald $Z=39.192, p<.001$ ). Hence, the between-participants variance is $15 \%$ of the total variance, meaning that in the null model the between-participants effect accounts for $15 \%$ of the total variance in buying intentions. ${ }^{3}$

In the final model (Table 3, Model 2), which includes product attributes and expected taste as fixed effects, the two variance components are 4.26 (within-participants variance, Wald $\mathrm{Z}=39.103, \mathrm{p}<$ .001 ) and 2.13 (between-participants variance, Wald $Z=11.105, \mathrm{p}<.001$ ).) The drop in the withinparticipants variance when controlling for product attributes and expected taste is a measure of how large a share of this variance is accounted for by the included variables. When controlling for product attributes and expected taste, the within-participants variance drops 4.58 (i.e., 8.84 minus 4.26 ) or $52 \% .{ }^{4}$ This value (.52) is an estimate of $\mathrm{R}^{2}$ (or explained variance) for our two-level random intercept model predicting buying intentions when controlling for expected taste and product attributes as fixed effects.

The fact that the within-participants variance component is large and significant in the final model means that there remains significant unexplained variance in buying intentions even after expected taste and product attributes are controlled. In this case, although within-participant variation is more important, since the between-participants variance component is statistically significant, we may conclude that buying intentions regarding chicken in general vary by individual. This means that observations are biased up or down depending on the individual participant and that a fixed-effects analysis of buying intentions ignoring the between-participants effect would hence have violated the assumption of independence of observations.

\section{Discussion}

\subsection{Summary of findings}

Our findings show that when the effects of product attributes are substantially attenuated after controlling for expected taste, the selected product attributes have the expected significant impact

\footnotetext{
${ }^{3}$ That this effect is significant reflects that buying intention scores for different chicken products tend to cluster by participants: buying intentions regarding two chicken products randomly selected from the same participant's nine evaluated products are more likely to be similar than buying intentions regarding two chicken products randomly selected from different participants.

${ }^{4}$ In the same way, we can calculate that controlling for product attributes alone accounts for $34 \%$ and controlling for expected taste alone for $40 \%$ of the within-participants variability in buying intentions.
} 
on expected taste. Consumers indeed seem to infer expectations about a chicken's taste from its weight, price, production method or information about the farmer and the rearing conditions. A low-weight chicken is expected to have inferior taste qualities. In contrast, the organic and free range claims, as well as the information about the farmer, are used as cues indicating superior taste qualities. It is interesting to notice that a high price level has no effect on expected taste, compared to a medium price, whereas low price has a negative impact. This means that a chicken that has a high price is not expected to taste better than a medium priced on, but that a low-priced chicken is expected to have inferior taste qualities.

In the case of the direct effect, where the mediator is not included in the analysis, we see that all the product attributes have a significant effect on buying intentions. Both organic and free range are strongly favoured over conventional chicken and both product characteristics lead to a significant and substantial increase in buying intentions, which is considerably bigger for organic than for free range. Higher price has the expected negative effect on buying intentions. Information about the farmer has a positive, but relatively weak effect on buying intentions. Both low weight and high weight have a negative impact on buying intentions, meaning that consumers' ideal chicken has a medium weight.

When expected taste is included in the analysis as a mediator, we first note that it has a highly significant effect on willingness to buy. The effect of low weight on buying intentions is completely mediated through expected taste. The effects of organic, free range and information about the farmer are partially mediated. We note that the direct effect is less than half of the total effect of the attributes on buying intentions. Thus we may infer that an important reason why consumers prefer chicken that are not too small, that are organic or free range, and that come with information about the farmer is that they use these attributes as cues to the taste quality of the chicken.

\subsection{Limitations}

The findings of our research are not representative for an entire population, due to the convenience sampling, which resulted (as desired) in an overrepresentation of heavy organic consumers compared to a representative sample of the adult Danish population. This means that findings based on the overall sample lead to conclusions that are mainly group or segment specific. However, this is not considered to be a major issue, due to the fact that organic chicken products have a low market share and thus appeal only to certain consumer segments.

Another limitation is that, by assuming fixed effects for product attributes and taste, we implicitly assume homogeneity of taste preferences across participants (i.e., same effects on all consumers). In future research this assumption might be relaxed, preferably in studies based on a large, representative sample of consumers. If heterogeneity of preferences were found in such a study, it would suggest the existence of different segments of consumers responding differently to product attributes, with obvious implications for market segmentation and marketing strategy.

\subsection{Implications}

In recent years, consumers have attached increasing importance to attributes that have no necessary relationship to the experienced product quality. Especially the way food is produced has become an important quality dimension, even in cases when it has no immediate bearing on the taste of the product (Brunsø, et al., 2002). Moreover, several previous studies have shown that taste is an important food quality dimension, some concluding that consumers have even higher taste expectations in the case of organic food. Our study confirms these assumptions. First, the outcome of the focus group studies was that taste is one of the most important quality dimensions in 
consumers' overall evaluation of chicken. Secondly, the results of the conjoint analysis have shown that taste expectations are higher for free range and organic chicken products compared to conventional chicken. Hence, our research confirms the fact that taste expectations play a major role in consumers' choice of chicken.

Previous research has shown that many consumers value process characteristics (such as organic production) in food products. There is, however, a lack of research into whether they are valued in their own right or because consumers use them as cues to eating quality. Our study tests the hypothesis that the impact of quality cues on consumers' willingness to buy organic vs. conventional or free-range chicken is partly mediated through taste expectations. The results of our analysis have shown that certain product attributes are used as quality cues and that they are associated with good taste. According to our findings, low weight and low price have a negative effect on expected taste, while above-average price does not influence taste expectations. The impact of all attributes on buying intentions is at least partly mediated through taste expectations the impact of low weight is completely mediated. Our research confirms the fact that taste expectations play a major role in consumers' choice of chicken and that part of the reason why credence characteristics (such as the organic claim) are important to consumers is that they are perceived as cues to superior taste quality.

The findings of our study have obvious implications for the marketing strategy of organic as well as conventional chicken products. Producers of conventional chicken need to put even more effort into persuading consumers about the tasting quality of their products than organic producers. Producers of organic chicken need a better differentiation strategy and to strive to provide quality where consumers need it. In new product development, we suggest that producers take into consideration what attributes have a direct impact on the expected as well as the experienced taste of the final product, given the fact that consumers make taste inferences based on the cues that are available. Regarding the communication strategy, the information that reaches consumers influence their buying decisions; therefore the message needs to emphasize quality dimensions that are important for the target consumers. This is especially important for meat products, because they cannot be evaluated completely until they are prepared or cooked. This means that consumers have to rely on the information they receive as cues to quality before actually purchasing the product.

Our study has demonstrated that product and process characteristics of chicken products are important for consumers in and off themselves, but not only that. Consumers use available information about product and process characteristics to make inferences about the product's eating quality. Hence, the effect of process characteristics, such as organic and free-range, on consumers' willingness to buy is party mediated by taste expectations. There is good reasons to assume that expected taste is one of the most important mediators of the relationship between product and process characteristics and willingness to buy, but it is not necessarily the only one. Hence, future research should identify other important mediators.

\section{Acknowledgements}

This study was conducted in connection with the SUMMER project, which is part of the Organic RDD programme and is coordinated by the International Center for Research in Organic Food Systems (ICROFS). The Danish AgriFish Agency, Ministry of Food, Agriculture and Fisheries is acknowledged for its financial support for the project. The authors also acknowledge the valuable contribution by Stine Bjerre and Jacob Overby, who collected the data and also designed the study under supervision by the second author. Finally, we thank Athanasios Krystallis, Joachim 
Scholderer, and two anonymous reviewers for valuable comments and suggestions to improve the manuscript.

\section{References}

Acebron, L. B., \& Dopico, D. C. (2000). The importance of intrinsic and extrinsic cues to expected and experienced quality: an empirical application for beef. Food Quality and Preferences, 11, 229238.

Aertsens, J., Verbeke, W., Mondelaers, K., \& Van Huylenbroeck, G. (2009). Personal determinants of organic food consumption: a review. [Review]. British Food Journal, 111(10), 1140-1167. Bandura, A. (1991). Social Cognitive Theory of Self-regulation. Organizational behavior and human decision processes, 50, 248-287.

Bandura, A. (1998). Health promotion from the perspective of social cognitive theory. Psychology and Health, 13, 623-649.

Baron, R. M., \& Kenny, D. A. (1986). The moderator-mediator variable distinction in social psychological research: conceptual, strategic, and statistical considerations. Journal of Personality and Social Psychology, 51, 1173-1182.

Becker, T. (2000). Consumer perception of fresh meat quality: a framework for analysis. British Food Journal, 102(3), 158-176.

Bredahl, L., \& Poulsen, C. S. (2002). Perceptions of pork and modern pig breeding among Danish consumers. Aarhus: Aarhus School of Business.

Brunsø, K., Fjord, T. A., \& Grunert, K. G. (2002). Consumers' food choice and quality perception: Aarhus School of Business, Aarhus University, MAPP Centre, Aarhus.

Conner, M., \& Armitage, C. J. (2002). The social psychology of food. Buckingham: Open University Press.

Costell, E., Tárrega, A., \& Bayarri, S. (2010). Food Acceptance: The Role of Consumer Perception and Attitudes. Chemosensory perception, 3(1), 42-50.

Grunert, K. (2005). Food quality and safety: consumer perception and demand. [Article]. European Review of Agricultural Economics, 32(3), 369-391.

Kennedy, O. B., Stewart-Knox, B. J., Mitchell, P. C., \& Thurnham, D. I. (2004). Consumer perceptions of poultry meat: a qualitative analysis. Nutrition and food science, 34(3/4), 122-129. Krystallis, A., Arvanitoyannis, I., \& Chryssohoidis, G. (2006). Is there a real difference between conventional and organic meat? Investigating consumers' attitudes towards both meat types as an indicator of organic meat's market potential. Journal of Food Products Marketing, 12(2), 47-78. Krystallis, A., \& Arvanitoyannis, I. S. (2006). Investigating the concept of meat quality from the consumers' perspective: The case of Greece. Meat Science, 72(1), 164-176.

Köster, E. P. (2009). Diversity in the determinants of food choice: A psychological perspective. Food quality and preference, 20(2), 70-82.

Köster, E. P., \& Mojet, J. (2007). Theories of food choice development Understanding Consumers of Food Products (pp. 93-124): CRC Press.

MacKinnon, D. P., Fairchild, A. J., \& Fritz, M. S. (2007). Mediation analysis. Annual Review of Psychology, 58, 593-614.

Magnusson, M. K., Arvola, A., Hursti, U.-K. K., berg, L., Sj, \& n, P.-O. (2001). Attitudes towards organic foods among Swedish consumers. British Food Journal, 103(3), 209-227.

McEachern, M. G., \& Willock, J. (2004). Producers and consumers of organic meat: A focus on attitudes and motivations. British food journal (1966), 106(6/7), 534-552.

Naspetti, S., \& Zanoli, R. (2009). Organic Food Quality and Safety Perception Throughout Europe. Journal of Food Products Marketing, 15(3), 249-266. 
Northen, J. R. (2000). Quality attributes and quality cues Effective communication in the UK meat supply chain. British Food Journal, 102(3), 230-245.

Scholderer, J., Nielsen, A. N., Bredahl, L., Caludi-Magnussen, C., \& Lindahl, G. (2004). Organic pork: consumer quality perceptions. [Project paper, MAPP, Denmark].

Stanton, J., Hughner, R. S., McDonagh, P., Prothero, A., \& Shultz, C. J. (2007). Who are organic food consumers? A compilation and review of why people purchase organic food. Journal of consumer behaviour, 6(2- 3), 94-110.

Stolz, H., Jahrl, I., Baumgart, L., \& Schneider, F. (2010). Sensory Experiences and Expectations of Organic Food. Results of Focus Group Discussions. Frick, Switzerland: Research Institute of Organic Agriculture (FiBL).

Thøgersen, J. (2009). Consumer Decision-Making with Regard to Organic Food Products

Traditional Food Production and Rural Sustainable Development. A European Challenge (pp. 173192). Surrey: Ashgate Publishing Limited.

Thøgersen, J., Jørgensen, A.-K., \& Sandager, S. (2012). Consumer Decision Making Regarding a “Green" Everyday Product. Psychology and Marketing, 29(4), 187-197.

Van Loo, E., Caputo, V., Nayga M. Rodolfo, J., Meullenet, J.-F., Crandall, P. G., \& Ricke, S. C. (2010). Effect of Organic Poultry Purchase Frequency on Consumer Attitudes Toward Organic Poultry Meat. Journal of Food Science, 75(7), 384-397.

Veale, R., \& Quester, P. (2009). Tasting quality: the roles of intrinsic and extrinsic cues. Asia Pacific Journal of Marketing and Logistics, 21(1), 195-207.

Zanoli, R., \& Naspetti, S. (2002). Consumer motivations in the purchase of organic food: A meansend approach. British food journal (1966), 104(8), 643-653.

Zeithaml, V. A. (1988). Consumer Perceptions of Price, Quality, and Value: A Means-End Model and Synthesis of Evidence. Journal of Marketing, 52(3), 2-22. 